\title{
In Vitro Evaluation of the Antimicrobial Activity of Nanosilver-Mineral Trioxide Aggregate against Frequent Anaerobic Oral Pathogens by A Membrane-enclosed Immersion Test
}

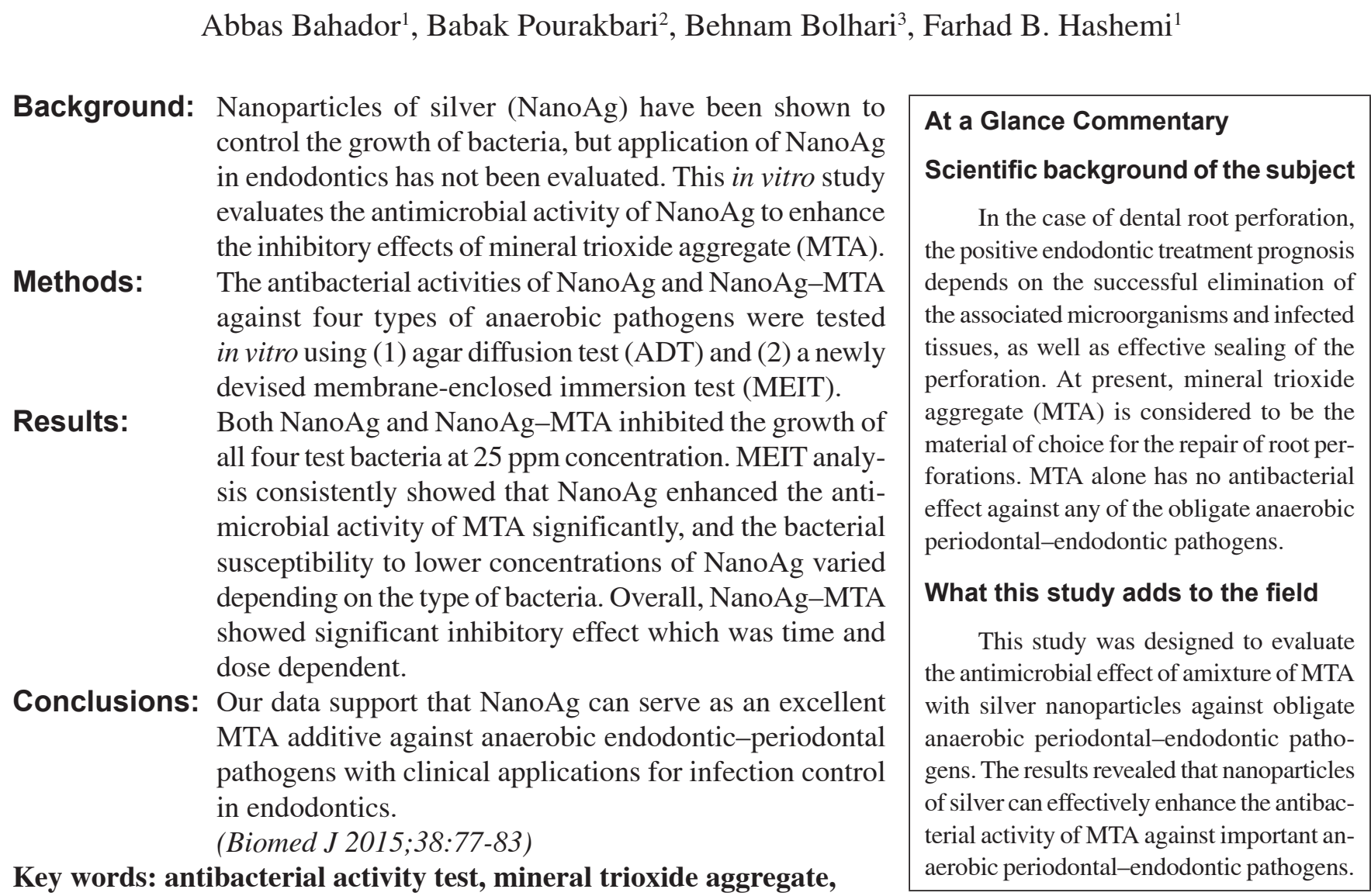
nanosilver

$\mathrm{C}$ Yontrol of microbial infection in periodontal and endodontic tissues is essential for the successful management of endodontic-periodontal lesions and external root resorption due to root canal perforation. ${ }^{[1-3]}$ The predominant bacterial genera responsible for endodontic-periodontal infections include Fusobacterium, Porphyromonas, Aggregatibacter, and Prevotella.$^{[4]}$ Mineral trioxide aggregate (MTA) is currently the material of choice for repairing root canal perforations and endodontic treatment. ${ }^{[5,6]}$ MTA is a water-based biocompatible material that promotes regeneration of surrounding tissues. ${ }^{[7-9]}$ Several studies have reported the antibacterial activity of MTA, but the results are inconsistent. ${ }^{[10-14]}$ Therefore, an additive that robustly enhances the inhibitory effects of MTA, while maintaining its biocompatibility, is highly desirable.

Nanoparticles of silver (NanoAg) have been reported to inhibit the bacterial growth of oral bacteria, ${ }^{[15,16]}$ as well

From the ${ }^{1}$ Department of Microbiology, School of Medicine, Tehran University of Medical Sciences, Tehran, Iran; ${ }^{2}$ Pediatrics Infectious Disease Research Center, School of Medicine, Tehran University of Medical Sciences, Tehran, Iran; ${ }^{3}$ Department of Endodontic, School of Dentistry, Tehran University of Medical Sciences, Tehran, Iran

Received: Jun. 18, 2013; Accepted: Mar. 11, 2014

Correspondence to: Dr. Farhad B. Hashemi, Department of Microbiology, School of Medicine, Tehran University of Medical Sciences, Iran. Keshavarz Blvd, Poursina Ave., 1416753955, Tehran, Iran. Tel: 98-021-8479531; Fax: 98-021-8955810; E-mail: fbh.ab.group2@gmail.com

DOI: $10.4103 / 2319-4170.132901$ 
as accelerate wound healing. ${ }^{[17-19]}$ However, the application of NanoAg in endodontics for the purpose of preventing infections in root canal perforations has not been explored. Therefore, the primary purpose of this study was to evaluate the antimicrobial activity of NanoAg-MTA mixture against several periodontal pathogens by several in vitro methods.

Agar diffusion test (ADT) remains the standard method for the in vitro evaluation of antimicrobial activity of substances. Since ADTs have certain limitations in evaluating materials pertaining to endodontics, ${ }^{[19]}$ a direct contact test (DCT) method and a modified DCT method have been developed to address the shortcomings of the ADT method. ${ }^{[20,21]}$ However, current DCT methods are not suitable for water-based repair materials such as MTA because fresh preparations disperse in aqueous media and form slurries, which can lead to sampling error. Consequently, we also describe a novel membrane enclosed immersion test (MEIT) method, devised to measure the antimicrobial activity of water-based endodontic cements, such as MTA, by directly exposing NanoAg-MTA to aqueous bacterial cultures.

\section{METHODS}

\section{Test microorganisms and growth conditions}

Lyophilized Aggregatibacter actinomycetemcomitans, Fusobacterium nucleatum, Porphyromonas gingivalis, and Prevotella intermedia (ATCC cultures 33384, 33277, 33270, and 49046, respectively, obtained from Rayen Biotechnology Co. Ltd, Tehran, Iran) were rehydrated in sBHI broth, which is brain heart infusion (BHI) broth (Merck, Darmstadt, Germany) supplemented with hemin $(5 \mu \mathrm{g} / \mathrm{ml})$ and vitamin $\mathrm{K}(1 \mu \mathrm{g} / \mathrm{ml})$, and incubated in an anaerobic atmosphere at $37 \mathrm{C}$ for $48 \mathrm{~h}$. Both items were purchased from Sigma-Aldrich (Steinheim, Germany).

For experiments requiring cultures on plates, cultures grown in sBHI broth were transferred onto sheep blood agar (BHK) plates containing Brucella agar (Merck) as the base medium, supplemented with hemin plus vitamin K. The sBHI broth and BHK agar cultures were used as described below.

\section{MTA and NanoAg-MTA preparations}

MTA (ProRoot ${ }^{\circledR}$ MTA; Maillfer, Dentsply, Switzerland) was mixed according to the manufacturer's recommendations by using $1 \mathrm{~g}$ of MTA powder for every $350 \mu \mathrm{l}$ of sterile water. Sterile distilled water was used to dilute the colloidal 10-nm-diameter NanoAg $0.1 \mathrm{mg} / \mathrm{ml}$ stock solution (Cat. No. PL-Ag-S10-10mg; PlasmaChem, GmbH, Berlin, Germany). The NanoAg samples of 25 parts per mil- lion (ppm), $12.5 \mathrm{ppm}$, and $6.25 \mathrm{ppm}$ were prepared by twofold serial dilutions of the NanoAg $0.1 \mathrm{mg} / \mathrm{ml}$ (100 ppm) stock solution.

Samples with various concentrations of NanoAg-MTA were prepared fresh prior to each experiment. Briefly, each gram of MTA powder was mixed with $350 \mu \mathrm{l}$ of $25 \mathrm{ppm}$, $12.5 \mathrm{ppm}$, and $6.25 \mathrm{ppm}$ preparations of NanoAg solution, on sterile glass slabs using a sterile spatula.

\section{Testing of antimicrobial activity}

\section{Determination of nanoag minimum inhibitory concentration}

To evaluate the antibacterial effects of NanoAg, the minimum inhibitory concentration (MIC) was determined by the broth dilution method. Prior to each experiment, $1 \mathrm{ml}$ of NanoAg stock solution was mixed in $1 \mathrm{ml} \mathrm{SBHI}$ broth medium and this mixture was diluted twofold serially in sBHI broth.

Fresh sBHI bacterial cultures, in the logarithmic growth phase (9-12 h old), were adjusted to a concentration of $10^{8}$ colony-forming units $(\mathrm{CFU}) / \mathrm{ml}$, as verified by both spectrophotometry $\left(\mathrm{OD}_{600}: 0.6-0.7\right)$ and colony counting. Bacterial cultures were diluted by transferring $100 \mu \mathrm{l}$ aliquots of the inocula into $900 \mu \mathrm{l}$ of the NanoAg preparations in sBHI broth. The test samples were incubated in an anaerobic atmosphere at $37 \mathrm{C}$ for $48 \mathrm{~h}$. The MIC was defined as the lowest concentration of NanoAg at which no visible bacterial growth was detected. Each experiment was performed three times, and the MIC of NanoAg observed in $>2$ experiments was reported. Inhibition of growth was verified by culture on BHK plates.

\section{Agar diffusion test}

Fresh bacterial colonies were suspended in SBHI broth and their turbidity was adjusted to $0.5 \mathrm{Mc}$ Farland standard, corresponding to approximately $1.5 \times 10^{8} \mathrm{CFU} / \mathrm{ml}$. To achieve a lawn of bacterial growth, $100 \mu \mathrm{l}$ of the bacterial suspension was evenly distributed onto the surface of a BHK plate using a sterile glass spreader. After inoculation, four wells were made, each $3 \mathrm{~mm}$ in diameter and $4 \mathrm{~mm}$ in depth, by removal of BHK agar with a puncher at equidistant points. The MTA and NanoAg-MTA mixtures were transferred into each well and gently pressed in place using an amalgam carrier. All plates were incubated at $37^{\circ} \mathrm{C}$ under anaerobic conditions. After $48 \mathrm{~h}$, the zones of inhibition (ZOIs) around each well were measured and recorded by a blinded assistant. The $3 \mathrm{~mm}$ diameter of each well was subtracted from the ZOI and reported. Results were averages of three independent assays, and all items were sterilized prior to use. Control wells were treated identically, except for bacterial inoculation. 


\section{Membrane enclosed immersion test}

Fresh colonies of test bacteria from BHK plates were suspended in sBHI broth, and bacterial density was visually adjusted to a turbidity of $0.5 \mathrm{McF}$ arland standard. The exact density (CFU/ml) of each suspension was verified on BHK plates (see above). MTA and NanoAg-MTA mixtures were prepared as previously described.

In a typical MEIT assay [Figure 1], either freshly prepared MTA or NanoAg-MTA paste (approximately $4 \mathrm{~mm} \times 4 \mathrm{~mm}$ ) was enclosed within a sterile microbiologic paper filter $(0.45 \mu \mathrm{m}$ Cat. No. HVLP04700, Millipore Co. Ltd, Tokyo, Japan). The membrane-wrapped mixture was then closed off at the top by sterile surgical thread, and immersed into the test wells of a 24-well plate containing $1 \mathrm{ml}$ of bacterial suspension. Adhesive tape was used to secure the wrapped membranes to the plate containing the test cultures [Figure 1]. The paper membrane served to prevent dispersion of MTA and formation of MTA slurry in the test wells. The direct physical interaction between the bacterial cells and NanoAg-MTA allowed for the exchange of soluble compounds between membrane-enclosed material and test bacteria. In addition, $1 \mathrm{ml}$ of the bacterial suspension in wells not containing MTA or NanoAg-MTA served as controls. The plates were then incubated at $37 \mathrm{C}$ under anaerobic conditions and then measured at $0.5,1,3,48$, and $72 \mathrm{~h}$ time points.

At each time point, $100 \mu \mathrm{l}$ of bacterial culture from each well was subcultured onto BHK plates, and bacterial growth and concentration was assessed. Throughout the MEIT experiments, cultures were checked for contamination by blind cultures on BHK plates, as well as visually examining for MTA slurry permeation into media. Several criteria were used to differentiate between contamination and the growth of test bacteria. These included the identity of the colonial morphology, quantity of growth, and the amount of time required for the organism to grow in the culture medium. At the time of sampling, no MTA slurry was observed in MEIT cultures, indicating that MTA remained mostly enclosed in the membrane wrap. All experiments were performed in triplicates, and bacterial concentrations were reported as mean \pm standard deviation (SD).

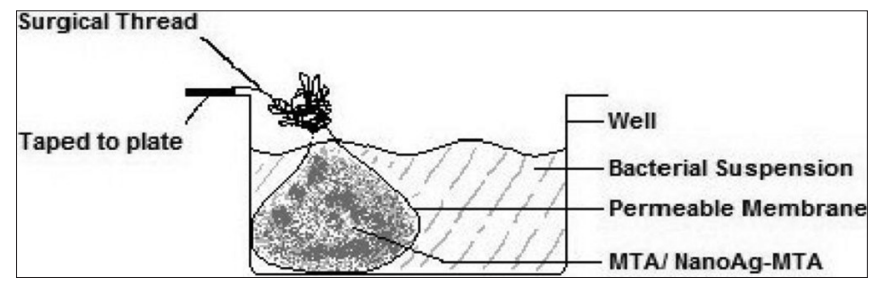

Figure 1: A schematic representation of the membrane-enclosed immersion test (MEIT). Samples of NanoAg-MTA, enclosed in membrane filters, were immersed into the wells of a 24-well plate, each containing $1 \mathrm{ml}$ of test bacterial suspension; and then were incubated for 0.5-48 h under anaerobic conditions.

\section{Statistical analysis}

The results of ADT and MEIT assays were analyzed using one-way analysis of variance (ANOVA) and Tukey's post hoc analysis using SPSS software (version 13; SPSS Inc., Chicago, IL, USA). In all analyses, the confidence level was set at $p<0.05$.

\section{RESULTS}

\section{NanoAg inhibition of growth of test microorganisms}

The MIC of NanoAg was $3.1 \mathrm{mg} / \mathrm{ml}$ (i.e. $3.1 \mathrm{ppm}$ ), which inhibited the growth of both A. actinomycetemcomitans and F. nucleatum (data not shown). However, the MIC of NanoAg against Po. gingivalis and Pr. intermedia was 6.25 and $12.5 \mathrm{ppm}$, respectively, indicating their lower sensitivity to NanoAg. Therefore, NanoAg was fourfold more potent in inhibiting the growth of $A$. actinomycetemcomitans and $F$. nucleatum than that of Pr. intermedia.

NanoAg-MTA inhibition of growth in test bacterial cultures

ADT analysis demonstrated that NanoAg-MTA inhibited the growth of all test bacteria. Table 1 shows that A. actinomycetemcomitans was the most sensitive bacterium tested, with susceptibility found at all the tested concentrations of NanoAg-MTA. On the other hand, Pr. intermedia showed the lowest sensitivity to NanoAg-MTA, which inhibited this species only at the $25 \mathrm{ppm}$ concentration, with a small ZOI of $4.0 \pm 2.0 \mathrm{~mm}$, and no inhibition at lower concentrations.

Moreover, with the exception of Pr. intermedia, $12.5 \mathrm{ppm}$ of NanoAg-MTA showed some level of inhibitory activity for all the test microorganisms. A. actinomycetemcomitans showed the largest ZOI $(11.0 \pm 2.0 \mathrm{~mm})$, followed by $F$. $n u$ cleatum $(7.0 \pm 1.0 \mathrm{~mm})$, and Po. gingivalis $(5.0 \pm 2.0 \mathrm{~mm})$. For the most resistant test bacterium, Pr. intermedia, the ZOIs around the $25 \mathrm{ppm}$ NanoAg-MTA wells were similar to the ZOI of wells containing MTA alone $(4.0 \pm 2.0 \mathrm{~mm})$.

Generally, the antimicrobial activity of 25 and

Table 1: Antimicrobial activity of NanoAg-MTA against microorganisms*

\begin{tabular}{lcccc}
\hline Microorganisms & \multicolumn{3}{c}{ Zone of inhibition $(\mathrm{mm})$} \\
\cline { 2 - 5 } & MTA & NanoAg-MTA concentration $(\mathrm{ppm})$ \\
\cline { 2 - 5 } & & 6.25 & 12.5 & 25 \\
\hline A. actinomycetemcomitans & $6 \pm 2$ & $9 \pm 1$ & $11 \pm 2$ & $16 \pm 1$ \\
F. nucleatum & $4 \pm 2$ & $4 \pm 2$ & $7 \pm 1$ & $11 \pm 1$ \\
Po. gingivalis & 0 & 0 & $5 \pm 2$ & $8 \pm 1$ \\
Pr. intermedia & 0 & 0 & 0 & $4 \pm 2$
\end{tabular}

*Expressed as zones of inhibition (mm). Numbers represent the mean $( \pm \mathrm{SD})$ of three independent experiments, and the cut-off was set at $3 \mathrm{~mm}$, Abbreviations: MTA: Mineral trioxide aggregate; NanoAg: Nanoparticles of silver 
12.5 ppm NanoAg-MTA was significantly higher than the activity of $6.25 \mathrm{ppm}$-treated cultures $(p<0.05)$. For $A$. actinomycetemcomitans $(6.0 \pm 2.0 \mathrm{~mm} \mathrm{ZOI})$ and $F$. nucleatum $(4.0 \pm 2.0 \mathrm{~mm} \mathrm{ZOI})$, there was no significant difference between the ZOIs of $6.25 \mathrm{ppm} \mathrm{NanoAg-MTA}$ treated wells versus the MTA-alone wells. Furthermore, wells with MTA alone showed no antimicrobial activity against $P$ r. intermedia and Po. gingivalis isolates. While MTA showed slight activity against A. actinomycetemcomitans and $F$. nucleatum, the ZOIs of MTA-alone wells were significantly smaller than those of wells with 12.5 and 25 ppm NanoAg-MTA.

Reduction of bacterial viability by immersed NanoAgMTA using MEIT method

Figure 2 shows that NanoAg-MTA markedly reduced the viability of bacterial cultures within hours of exposure. On the contrary, MTA alone showed negligible antibacterial activity against all four test bacteria. Overall, the MEIT results were consistent with the results of our ADT analysis.
Throughout the MEIT analysis, the growth of cultures treated with NanoAg-MTA was compared to the growth of test microorganisms in untreated cultures which showed about 50-1000 fold increase over a $48 \mathrm{~h}$ test period [Figure 2].

MEIT analysis demonstrated that $25 \mathrm{ppm}$ NanoAgMTA inhibited the growth of all test bacteria by $60-100 \%$. As shown in Figure 2A-C, exposure to $25 \mathrm{ppm}$ NanoAg-MTA for $3 \mathrm{~h}$ had a marked bactericidal effect on Po. gingivalis, as well as A. actinomycetemcomitans and $F$. nucleatum cultures, with a complete decline in culture viability. After a $1 \mathrm{~h}$ exposure, $25 \mathrm{ppm}$ NanoAg-MTA reduced the culture viability of $A$. actinomycetemcomitans and $F$. nucleatum by $58 \%$ and $38 \%$, respectively. On the other hand, $25 \mathrm{ppm}$ NanoAg-MTA reduced the viability of Pr. intermedia by 32-60\%, suggesting a bacteriostatic effect on Pr. intermedia cultures during 3-48 h of incubation [Figure 2D]. Most notably, at all time points of the MEIT assay, $25 \mathrm{ppm}$ NanoAg-MTA showed a higher inhibitory activity than

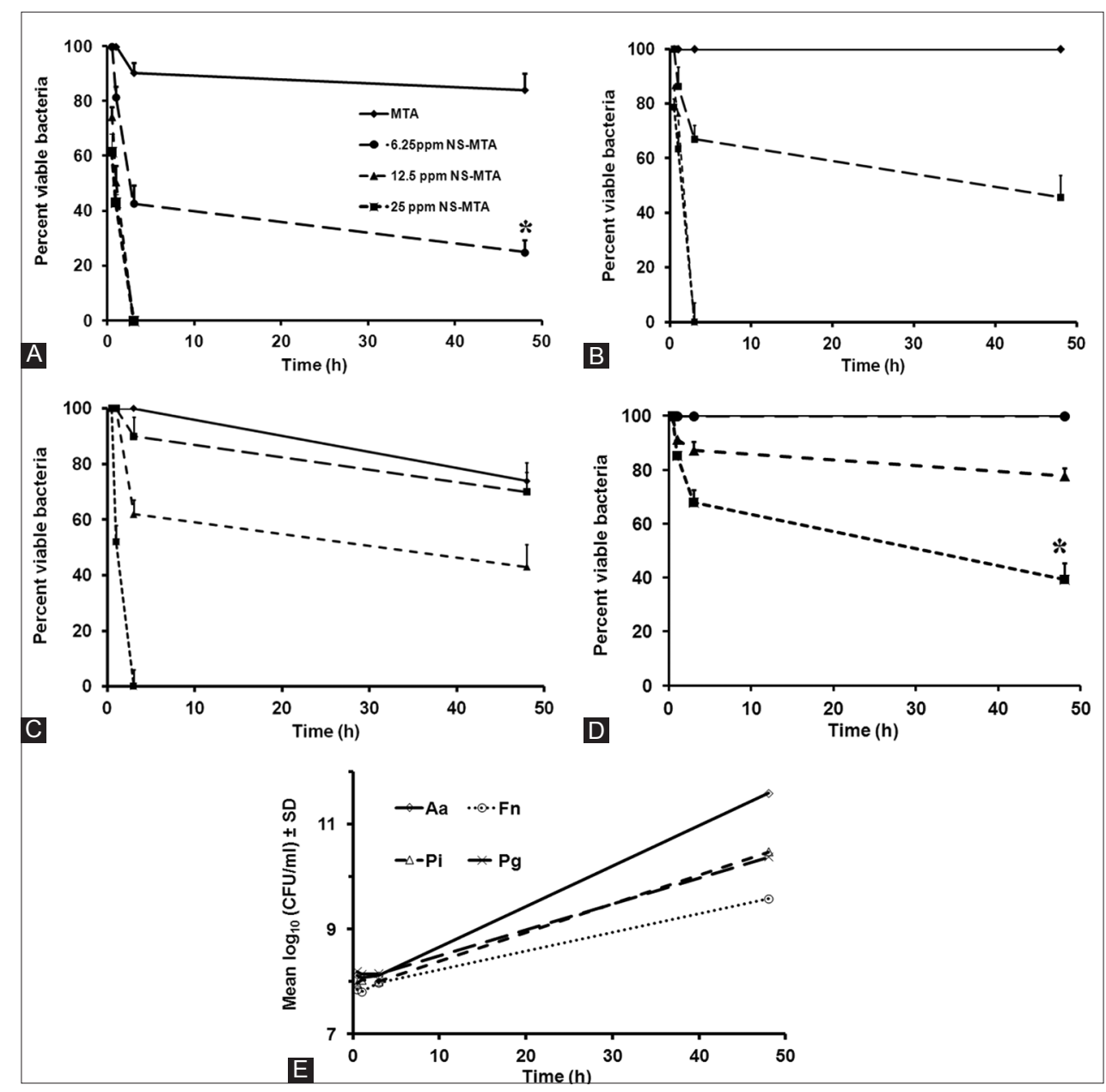

Figure 2: Effect of NanoAg-MTA on bacterial viability using MEIT method. The antibacterial effects of NanoAg-MTA against: (A) A. actinomycetemcomitans (Aa), (B) F. nucleatum (Fn), (C) Po. gingivalis (Pg), and (D) Pr. intermedia (Pi) were assessed. Bacterial cultures were treated with the indicated concentrations of NanoAg-MTA for up to $48 \mathrm{~h}$. Mean percent reduction in culture viability was calculated as compared to untreated cultures and (E) the bacterial growth curve of untreated sBHI broth cultures over a $48 \mathrm{~h}$ period. Mean percent viability of each culture was calculated by colony counting. 
MTA alone which did not inhibit the growth of any test culture. The differences between the antibacterial effects of samples containing $25 \mathrm{ppm}$ NanoAg-MTA versus those with MTA alone were statistically significant $(p<0.05)$. Furthermore, extending the incubation period of MEIT assay to $72 \mathrm{~h}$ did not significantly change the MEIT culture viability results (data not shown).

In addition, $12.5 \mathrm{ppm}$ NanoAg-MTA had a strong bactericidal effect on both A. actinomycetemcomitans and F. nucleatum cultures after $3 \mathrm{~h}$ of incubation [Figure $2 \mathrm{~A}$ and B]. However, the same level of NanoAg-MTA showed only slight antibacterial effects on Po. gingivalis and $P r$. intermedia cultures [Figure $2 \mathrm{C}$ and D]. After a $48 \mathrm{~h}$ exposure, $12.5 \mathrm{ppm}$ NanoAg-MTA reduced the viability of $P o$. gingivalis and Pr. intermedia by $38 \%$ and $12 \%$, respectively, with lesser antibacterial effect at earlier time points. The antibacterial activity of $6.25 \mathrm{ppm}$ NanoAg-MTA ranged from modest to none. Cultures of A. actinomycetemcomitans and $F$. nucleatum showed the highest susceptibility to $6.25 \mathrm{ppm}$ of NanoAg-MTA that reduced the bacterial viability by $63 \%$ and $54 \%$, respectively. Furthermore, 6.25 ppm NanoAg-MTA did not inhibit the growth of Po. gingivalis or Pr. intermedia cultures at any of the tested time points, similar to those treated with MTA alone.

At 25 and 12.5 ppm NanoAg-MTA, the antibacterial activity against $A$. actinomycetemcomitans and $F$. nucleatum cultures was time-dependent, such that extending the length of NanoAg-MTA exposure from 1 to $3 \mathrm{~h}$ enhanced the inhibitory effects of NanoAg-MTA on these microorganisms. Also, increasing the exposure period of Po. gingivalis cultures to $25 \mathrm{ppm}$ NanoAg-MTA by $2 \mathrm{~h}$ (from $1 \mathrm{~h}$ to $3 \mathrm{~h}$ ) led to a significant reduction in the number of viable bacteria in Po. gingivalis cultures [Figure 2C]. Although the $1 \mathrm{~h}$ and $3 \mathrm{~h}$ time points showed reduction in the viability of $P r$. intermedia cultures treated with 25 ppm NanoAg-MTA, these reductions were not significant [Figure 2D].

\section{DISCUSSION}

Perforation of the dental root creates non-natural connections between the root canal system and the periodontal ligament. Such perforations can be complications resulting from over instrumentation during endodontic procedures, internal or external resorption, or caries invading through the floor of the pulp chamber. Of all root perforations, $47 \%$ were found to be related to endodontic procedures and $53 \%$ were related to prosthodontic treatment. ${ }^{[22]}$ In perforations of root where periodontal disease is present, major periodontal pathogens such as Po. gingivalis, F. nucleatum, A. actinomycetemcomitans, and Pr. intermedia could be accompanying endodontic infections due to the periodontic-endodontic interrelationships. ${ }^{[23]}$ Periodontal disease is a frequently prevalent oral health problem with wide variations in severity and prevalence across different geographic areas. In adult periodontitis, Po. gingivalis, F. nucleatum, A. actinomycetemcomitans and Pr. intermedia were detected in $75 \%, 50 \%, 36 \%$, and $35 \%$ of thediseased sites, respectively. ${ }^{[24]}$ Endodontic diseases (pulpitis and periradicular infections) are polymicrobial and predominantly caused by strict anaerobic bacteria. ${ }^{[25]}$ The isolation rate of Gram-negative anaerobes in endodontic infections varies up to $85 \%$. In endodontics, F. nucleatum is the most commonly found Gram-negative anaerobe, followed by Po. gingivalis, Pr. Intermedia, and A. actinomycetemcomitans (85\%, 65\%, $62 \%$, and $33 \%$, respectively). ${ }^{[24]}$

To prevent infection after repairing a perforated root canal, a biocompatible obturation material with robust antimicrobial activity is highly desirable. We explored the potential of silver nanoparticles (NanoAg) applied as an additive to MTA toward the prevention of endodontic treatment failure. In the present study, we observed that NanoAg enhances the antimicrobial activity of MTA, and NanoAg-MTA can be effective against four periodontal/ endodontic pathogens. However, whether MTA retains its biocompatibility after mixing with NanoAg remains to be determined. However, a recent in vivo study using a concentration of $23 \mathrm{ppm}$ NanoAg showed a mild tissue reaction to NanoAg preparation. ${ }^{[26]}$ Although the NanoAg application proposed in our study differs from that in the report of Gomes-Filho et al., ${ }^{[26]}$ which describes the biocompatibility of $23 \mathrm{ppm}$ NanoAg dispersion as an evidence for potential NanoAg application as a root canal irrigant, the mild tissue reaction observed over $24 \mathrm{~h}$ lends support to potential application of NanoAg (25-ppm) as an obturation material.

The results of this study are consistent with a recent report in which the addition of silver zeolite (SZ) enhanced the antimicrobial activity of MTA. ${ }^{[14]}$ However, NanoAg-MTA has a higher antibacterial activity than SZ against some microorganisms. For instance, unlike SZ, NanoAg-MTA inhibited the growth of Pr. intermedia. ${ }^{[14]}$ We speculate that NanoAg has a higher bactericidal activity than the SZ preparation. Alternatively, our novel MEIT assay has a higher sensitivity in assessing the antibacterial activity than the ADT method used in the SZ report. The latter explanation highlights the potential utility of the MEIT assay as a practical alternative for testing the antibacterial activity of a broad range of endodontic materials. Notably, our finding that MTA had no antibacterial effect against any anaerobic bacteria is similar to previous reports. ${ }^{[27,28]}$ Since MTA's generation of reactive oxygen species (ROS), responsible in part for its antimicrobial effect is decreased under anaerobic conditions, it has been proposed that MTA may not produce adequate ROS to inhibit certain bacterial strains. ${ }^{[29,30]}$ We acknowledge that further evaluation of the antibacterial activity of NanoAg-MTA against additional 
common endodontic pathogens, such as Enterococcus faecalis, will strengthen our study findings, since E. faecalis is the most frequently recovered bacteria from failed endodontic treatment cases. ${ }^{[31]}$

While DCT is suitable for materials less likely to disperse in aqueous media, ${ }^{[19]}$ our novel MEIT assay has successfully measured the antibacterial activity of a water-based NanoAgMTA root end material. The absence of MTA contamination of aqueous media throughout the $72 \mathrm{~h}$ period supports the use of MEIT assay for direct assessment of antibacterial activity of any water-based, and/or oil-based, endodontic material in liquid cultures. The effectiveness of MEIT in preventing permeation of MTA slurry, which may cause errors in bacterial counts by mixing with samples, is consistent with studies which show that about $90 \%$ of MTA particles would not pass through the pores of $0.45-\mu \mathrm{M}$ membrane. ${ }^{[32]}$

Despite the afore-mentioned advantages, the MEIT assay is relatively laborious and time consuming. Thus, efforts to modify and streamline this assay are underway. Moreover, in light of the importance of microbial biofilm formation as a cause of bacterial persistence in root canals, ${ }^{[33]}$ the use of a bacterial suspension model can be a limitation to MEIT assay and this study. To address this issue, further studies using a modified MEIT assay that incorporates an endodontic biofilm model should be considered.

\section{Conclusions}

Our data support that NanoAgcan effectively enhance the antibacterial activity of MTA against important anaerobic periodontal-endodontic pathogens. To fully assess the viability of NanoAg-MTA, future studies will focus on gauging the physical properties of NanoAg-MTA, such as setting and working time. Moreover, since addition of NanoAg might change the efficiency of MTA in sealing root canal perforations, a thorough assessment of the sealing efficiency of NanoAg-MTA using a leakage model is necessary prior to clinical application of NanoAg-MTA. Also, considering that MTA usually remains in patients many years after endodontic treatment, the long-term effects of adding NanoAg to MTA merit investigation.

\section{REFERENCES}

1. Zehnder M, Gold SI, Hasselgren G. Pathologic interactions in pulpal and periodontal tissues. J Clin Periodontol 2002;29:663-71.

2. Mohammadi Z, Palazzi F, Giardino L, Shalavi S. Microbial biofilms in endodontic infections: an update review. Biomed $\mathrm{J}$ 2013;36:59-70.

3. Shokri A, Mortazavi H, Salemi F, Javadian A, Bakhtiari H, Matlabi H. Diagnosis of simulated external root resorption using conventional intraoral film radiography, CCD, PSP, and CBCT: A comparison study. Biomed J 2013;36:18-22.
4. Pereira CV, Stipp RN, Fonseca DC, Pereira LJ, Höfling JF. Detection and clonal analysis of anaerobic bacteria associated to endodontic-periodontal lesions. J Periodontol 2011;82:1767-75.

5. Mente J, Hage N, Pfefferle T, Koch MJ, Geletneky B, Dreyhaupt J, et al. Treatment outcome of mineral trioxide aggregate: Repair of root perforations. J Endod 2010;36:208-13.

6. Lodiene G, Kleivmyr M, Bruzell E, Ørstavik D. Sealing ability of mineral trioxide aggregate, glass ionomer cement and composite resin when repairing large furcal perforations. Br Dent J 2011;12:216-7.

7. Pistorius A, Willershausen B, Briseño Marroquin B. Effect of apical root-end filling materials on gingival fibroblasts. Int Endod J 2003;36:610-5.

8. Yoshimine $\mathrm{Y}$, Ono $\mathrm{M}$, Akamine A. In vitro comparison of the biocompatibility of mineral trioxide aggregate, 4META/MMA-TBB resin, and intermediate restorative material as root-end-filling materials. J Endod 2007;33:1066-9.

9. Baek SH, Lee WC, Setzer FC, Kim S. Periapical bone regeneration after endodontic microsurgery with three different root-end filling materials: Amalgam, SuperEBA, and mineral trioxide aggregate. J Endod 2010;36:1323-5.

10. Stowe TJ, Sedgley CM, Stowe B, Stowe B, Fenno JC. The effects of chlorhexidine gluconate $(0.12 \%)$ on the antimicrobial properties of tooth-colored ProRoot mineral trioxide aggregate. J Endod 2004;30:429-31.

11. Holt DM, Watts JD, Beeson TJ, Kirkpatrick TC, Rutledge RE. The anti-microbial effect against enterococcus faecalis and the compressive strength of two types of mineral trioxide aggregate mixed with sterile water or $2 \%$ chlorhexidine liquid. J Endod 2007;33:844-7.

12. Zhang H, Pappen FG, Haapasalo M. Dentin enhances the antibacterial effect of mineral trioxide aggregate and bioaggregate. J Endod 2009;35:221-4.

13. Parirokh $\mathrm{M}$, Torabinejad $\mathrm{M}$. Mineral trioxide aggregate: A comprehensive literature review-Part I: Chemical, physical, and antibacterial properties. J Endod 2010;36:16-27.

14. Odabaş ME, Cinar C, Akça G, Araz I, Ulusu T, Yücel H. Short-term antimicrobial properties of mineral trioxide aggregate with incorporated silver-zeolite. Dent Traumatol 2011;27:189-94.

15. Ahn SJ, Lee SJ, Kook JK, Lim BS. Experimental antimicrobial orthodontic adhesives using nanofillers and silver nanoparticles. Dent Mater 2009;25:206-13

16. Zhao L, Wang H, Huo K, Cui L, Zhang W, Ni H, et al. Antibacterial nano-structured titania coating incorporated with silver nanoparticles. Biomaterials 2011;32:5706-16.

17. Atiyeh BS, Costagliola M, Hayek SN, Dibo SA. Effect of silver on burn wound infection control and healing: Review of the literature. Burns 2007;33:139-48

18. Sibbald RG, Contreras-Ruiz J, Coutts P, Fierheller M, Rothman A, Woo K. Bacteriology, inflammation, and healing: A study of nanocrystalline silver dressings in chronic venous leg ulcers. Adv Skin Wound Care 2007;20:549-58.

19. Tian J, Wong KK, Ho CM, Lok CN, Yu WY, Che CM, et al. Topical delivery of silver nanoparticles promotes wound healing. Chem Med Chem 2007;2:129-36.

20. Meryon SD, Johnson SG. The modified model cavity method for assessing antibacterial properties of dental restorative materials. 
J Dent Res 1989;68:835-9.

21. Weiss EI, Shalhav M, Fuss Z. Assessment of antibacterial activity of endodontic sealers by a direct contact test. Endod Dent Traumatol $1996 ; 12: 179-84$

22. Tsesis I, Rosenberg E, Faivishevsky V, Kfir A, Katz M, Rosen E. Prevalence and associated periodontal status of teeth with root perforation: A retrospective study of 2002 patients' medical records. J Endod 2010;36:797-800.

23. Rupf S, Kannengiesser S, Merte K, Pfister W, Sigusch B, Eschrich K. Comparison of profiles of key periodontal pathogens in periodontium and endodontium. Endod Dent Traumatol 2000;16:269-75.

24. Joshi VM, Vandana KL. The detection of eight putative periodontal pathogens inadult and rapidly progressive periodontitis patients: an institutional study. Indian J Dent Res 2007;18:6-10.

25. Baumgartner JC, Siqueira JF Jr, Xia T, Róças IN. Geographical differences in bacteria detected in endodontic infections using polymerase chain reaction. J Endod 2004;30:141-4.

26. Gomes-Filho JE, Silva FO, Watanabe S, Cintra LT, Tendoro KV, Dalto LG, et al. Tissue reaction to silver nanoparticles dispersion as an alternative irrigating solution. J Endod 2010;36:1698-02 .
27. Torabinejad M, Hong CU, Pitt Ford TR, Kettering JD. Antibacterial effects of some root end filling materials. J Endod 1995;21:403-6.

28. Estrela C, Bammann LL, Estrela CR, Silva RS, Pecora JD. Antimicrobial and chemical study of MTA, PC, calcium hydroxide paste, Sealapex and Dycal. Braz Dent J 2000;11:3-9.

29. Cabiscol E, Tamarit J, Ros J. Oxidative stress in bacteria and protein damage by reactive oxygen species. Int Microbiol 2000;3:3-8.

30. Ribeiro CS, Scelza MF, Hirata Junior R, Buarque de Oliveira LM. The antimicrobial activity of gray-colored mineral trioxide aggregate (GMTA) and white-colored MTA (WMTA) under aerobic and anaerobic conditions. Oral Surg Oral Med Oral Pathol Oral Radiol Endod 2010;109:109-12.

31. Poptani B, Sharaff M, Archana G, Parekh V. Detection of Enterococcus faecalis and Candida albicans in previously root-filled teeth in a population of Gujarat with polymerase chain reaction. Contemp Clin Dent 2013;4:62-6.

32. Komabayashi T, Spångberg LS. Particle size and shape analysis of MTA finer fractions using PC. J Endod 2008;34:709-11.

33. Chaxvez de Paz LE. Redefining the persistent infection in root canals: possible role of biofilm communities. J Endod 2007;33:652-62. 